\title{
Optimized synthesis of a microwave equalizer for matching a high performance small antenna
}

\author{
D.Martínez, F.Seyfert, M.Olivi \\ INRIA Sophia-Antipolis Méditerranée \\ 2004 rte des Lucioles \\ 06902 Sophia Antipolis, FRANCE
}

\author{
J.Sence, S.Bila, F.Torrès, \\ T.Monédière, B.Jarry, A. Périgaud \\ XLIM UMR7252 Université de Limoges/CNRS \\ 123 av.Albert Thomas \\ 87060 Limoges, FRANCE
}

\author{
B.Frigui, R.Chantalat \\ CISTEME \\ 12 rue Gémini \\ 87068 Limoges, FRANCE
}

\begin{abstract}
A dual-band RHCP (right-hand circular polarization) microstrip patch antenna is presented for its implementation on a GNSS receiver covering the bands L2, E6 and L1. To improve the total efficiency at the band edges, a dualband equalized is designed by implementing a dual-band filter matched to the complex impedance of the antenna. This filter is composed of two single-band filters, each of them designed to maximize the minimum efficiency in the correspondent band, connected together with input and output junctions. Moreover a microstrip prototype for the dual-band equalizer is designed and fullwave simulations show the equalized efficiency validating the theoretical results.
\end{abstract}

\section{INTRODUCTION}

The deployment of electronic systems devoted to communication, localization, or supervision has to answer an increasing demand in terms of integration density and flexibility, both in frequency and coverage. The flexibility in frequency can be simultaneous with multi-band devices or selective with reconfigurable devices. The flexibility of coverage allows directing the beam through the target in order to optimize the link budget.

The increase of both efficiency and compactness requires co-designing elementary functions (antenna, filter, amplifier...), which allows reducing interconnection stages and taking into account the interactions between individual elements [1], [2], [3], leading to optimized sub-systems. In the frame of this work, the demonstration will be given with the co-design of dual band filters together with a RHCP (right-hand circular polarization) dual-band antenna for improving the total efficiency of a receiver for GNSS (Global Satellite Navigation System) radio navigation systems.

\section{DuAl-BAND RHCP ANTENNA}

The antenna design is described in details in [4]. The structure, depicted in Fig. 1. consists of a microstrip patch antenna with two orthogonal excitation ports and four slots, providing a dual-band circular polarization behaviour when the ports are combined with 90 offsets. The 2-port antenna is designed considering severe specifications in terms of gain, axial ratio and surface occupation. In order to fulfil all specifications, it comes difficult to match the antenna over the two passbands. The scattering parameters are given in Fig. 2

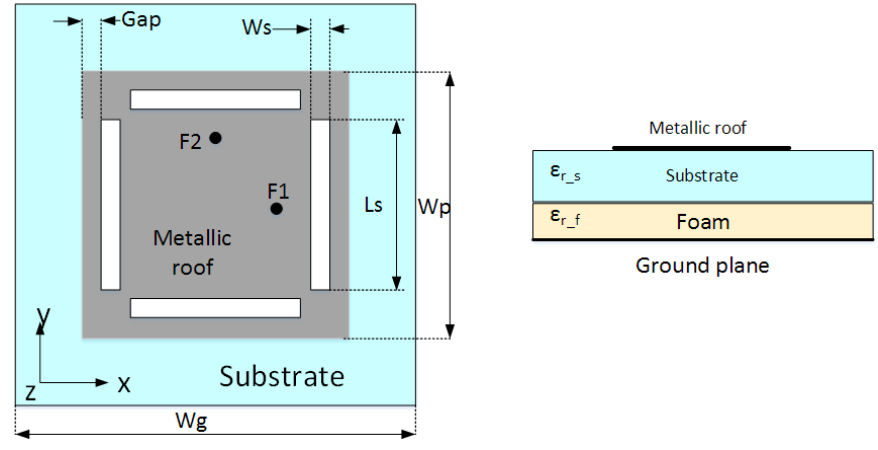

Fig. 1. Dual-band RHCP antenna integrated a microstrip slotted patch

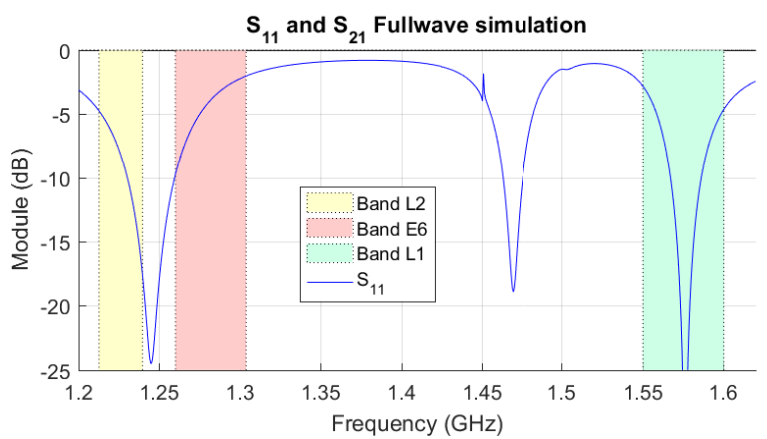

Fig. 2. Simulated scattering parameters of the dual-band RHCP (2-port) antenna.

The antenna is connected to a coupler for combining the 2 excitation ports, as shown in Fig. 3 Since the antenna is not perfectly matched in the two passbands (L2 + E6 is the first passband and L1 is the second passband), a non-negligible part of the signal is dissipated in the load connected to the isolated port. Consequently, the total efficiency is degraded in the passband edges as shown in Fig. 4. In order to improve the total efficiency, matching filters have to be designed and optimized with respect to antenna scattering parameters.

\section{A COMPLEX EQUALIZATION PROBLEM}

The problem of matching is stated from the de-embedding point of view. It consist on the design of the global system equalizer + load directly with a set of conditions to ensure 


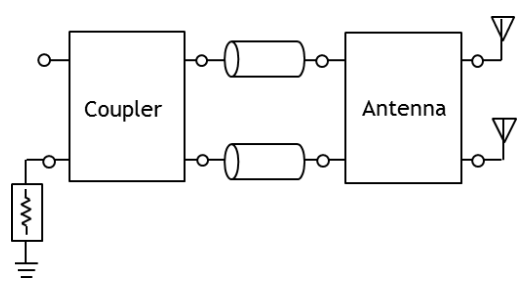

Fig. 3. Schematic of the RHCP antenna connected to a hybrid coupler for combining the two ports with 90 phase difference.
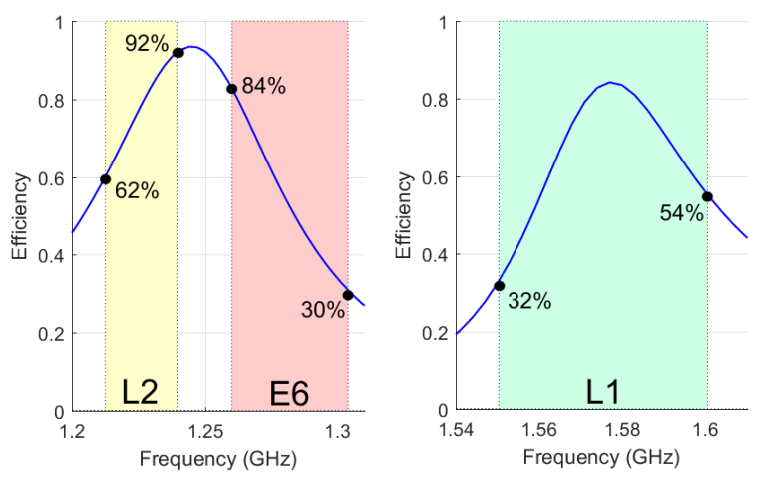

Fig. 4. Total efficiency of the dual-band RHCP antenna connected to the hybrid coupler.

that the load is included in such a system and can be extracted afterwards [5], [6]. To simplify the design, we perform a first order rational approximation of the antenna in each band. For a load of degree 1 , these conditions become just a constraint on the integral of the reflection coefficient (in logarithmic form). In Fig. 6 we can see an example of equalization for a load of degree 1 (the surface under both lines must be the same). For instance, as in the previous example, the reflection of global system (dashed line in Fig. 6) is computed as:

$$
\left|S_{11}\right|^{2}=\frac{|T|^{2}+\alpha^{2}}{|T|^{2}+\beta^{2}}
$$

while the transmission coefficient takes the form:

$$
\left|S_{21}\right|^{2}=\frac{\beta^{2}-\alpha^{2}}{|T|^{2}+\beta^{2}}
$$

where $T$ is a reference filtering function (here we take just the Tchebyshev polynomial) of the desired degree and $\alpha, \beta$ are positive constant with $\beta \geq \alpha$.

It can be proved that $\beta=\beta(\alpha)$ is a function that provides the right surface under the curve of return losses for every $\alpha$. Moreover for any filtering function $T$, the level of return losses in the passband has only one maximum with respect to $\alpha$.

By taking $T$ as the Tchebyshev polynomial of degree $\mathrm{N}$, and being $s$ the desired surface, we obtain:

$$
\begin{aligned}
\beta(\alpha) & =\sinh \left(N \operatorname{arcsinh}\left(\sinh \left(\frac{\operatorname{arcsinh}(\alpha)}{N}\right)+H\right)\right) \\
H & =\frac{s}{2 \pi} \sin \left(\frac{\pi}{2 N}\right)
\end{aligned}
$$

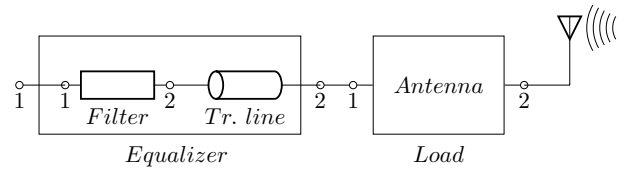

Fig. 5. Equalization chain.

The return losses obtained after equalization can be computed as:

$$
\begin{aligned}
|R L|^{2} & =\frac{\cosh ^{2}(N \operatorname{arcsinh}(x))}{\cosh ^{2}(N \operatorname{arcsinh}(x-H))} \\
x & =H+\sinh \left(\frac{1}{N} \operatorname{arcsinh}(\alpha)\right)
\end{aligned}
$$

Finally, after the extraction of the load the equalized filter is obtained along with the length of the transmission line at the output port (Fig. 5). It should be noted that only the equalizer for the first polarization is shown here along with the part of the antenna corresponding to the first polarization. Nevertheless the antenna is symmetrical and the cross-polarization can be neglected. Thus another identical equalizer would be required for the second polarization.

\section{Gain equalization}

With the aim to compute the effective transmission of the system when losses are considered, we work with a 2-ports model of the antenna where the second port represent the transmitted signal regarding the first polarization (efficiency). The transmission coefficient of the filter cascaded with the 2-ports antenna takes the following expression:

$$
S_{21}^{C}=\frac{S_{21}^{F} S_{31}^{A}}{1-S_{22}^{F} S_{11}^{A}}
$$

where the scattering parameters $S_{i j}^{A}$ refers to the antenna and the parameters $S_{i j}^{F}$ to the equalizer including the transmission line at the output port.

The objective now in is to maximize the transmission $\left(S_{21}^{C}\right)$ with a given requirement on the rejection in the stopband. With the condition over the rejection, the problem remains:

$$
\underset{S^{F}}{\operatorname{Max}}\left(\underset{f \in I}{\operatorname{Min}_{i}}\left|S_{21}^{C}\right|-\gamma \sum_{i}\left|S_{21}^{C}\left(f_{s i}\right)\right|\right)
$$

where $I$ represents the passband, $f_{s i}$ the frequencies to reject and $\gamma$ a positive weight. This is a local MaxMin optimization that converges extremely fast if the equalizing filter for the first order approximation of the load is taken as initial point.

\section{DUAL-BAND FILTER}

In order to perform a dual-band equalization, a dual-band filter is designed by implementing a duplexed solution and performing a fix point algorithm over both channels. In each iteration of the fix-point algorithm, the equalizer of one of the channels is designed by taking as load the system composed by the junction, the antenna and the equalizer of the other 


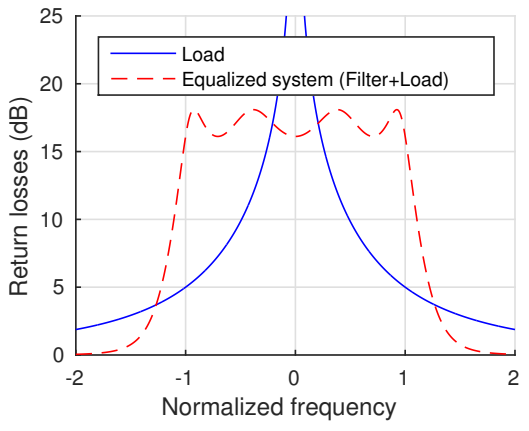

Fig. 6. Load of degree 1 along with the result of the equalization.

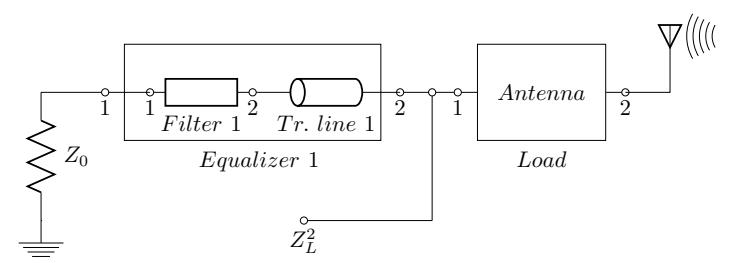

(a) Load for the second channel $\left(Z_{L}^{2}\right)$.

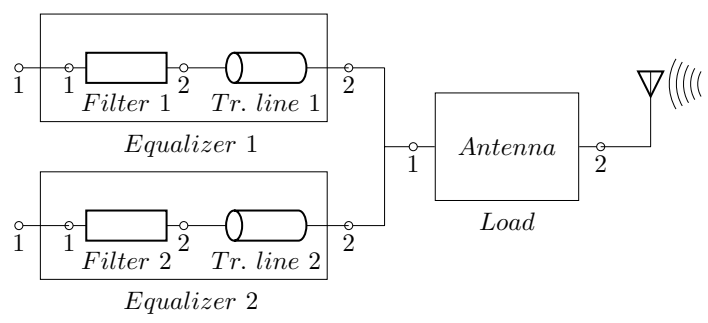

(b) Diplexed equalizers after the fix-point algorithm.

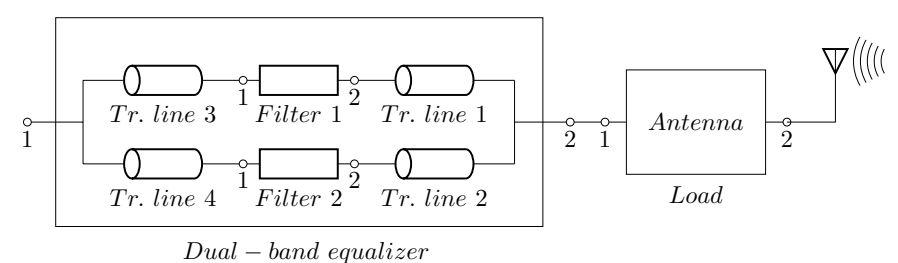

(c) Dual-band equalization chain.

Fig. 7. Different stages in the design of the dual-band equalizer.

channel. Below we show a detailed implementation of this fix-point algorithm:

1) Compute the single-band equalized for the first band (Fig. 5).

2) The impedance in the second band of the first channel equalized shunt with the antenna become the load for the second channel $\left(Z_{L}^{2}\right.$ in Fig. 7a). Then a new equalizer is made for the second band.

3) This process is iterated until the changes in each band become negligible. We obtain a diplexer with two singleband equalizers (Fig. 7b).

Finally, in order to compute the input junction, since the

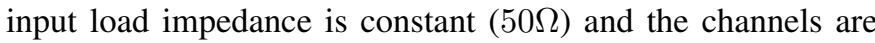
not too close to each other, we can just compute the length of the input lines such as at the centre of each band, the adjacent channel is seen as an open circuit. Fig. 7c shows the final schematic for the dual-band equalizer where the coupling matrices of both filter are shown in (9) and (10) and the lengths of the transmission lines 1 and 2 are $17 \mathrm{~mm}$ and $46 \mathrm{~mm}$ respectively.

$$
\begin{aligned}
M_{1} & =\left[\begin{array}{cccc}
0 & 0.9142 & 0 & 0 \\
0.9142 & -0.0570 & 0.9630 & 0 \\
0 & 0.9630 & 1.0883 & 0.7070 \\
0 & 0 & 0.7070 & 0
\end{array}\right] \\
M_{2} & =\left[\begin{array}{cccc}
0 & 1.0430 & 0 & 0 \\
1.0430 & -0.3964 & 1.0533 & 0 \\
0 & 1.0533 & -1.0144 & 0.8295 \\
0 & 0 & 0.8295 & 0
\end{array}\right]
\end{aligned}
$$

\section{Physical StRucture Synthesis}

The filter synthesis indicates that, to achieve the dual-band filtering function, it is necessary to implement two duplexed two poles filters. The strongest constraint in this study is the relative bandwidth of the two combined filters which is approximately $27 \%$. In order to build these two filters, it is necessary to choose a technology and a topology adapted to wide band filtering. The strongest constraint in this study is the relative bandwidth of the two combined filters which is approximately 27\%. An usual solution is then planar quarter wavelength resonators filter. Indeed, this type of filter presents the advantage to exhibit strong couplings meanwhile it does not resonate until 3 times the initial resonant frequency.

The main drawback of this type of structure is that it requires electric wall at one of its ends, that is generally ensured by one or more via(s) connected to ground.

However, this point does not require any additional manufacturing step to be addressed since vias are already necessary to build the coplanar feeding-lines of the antenna.

Each filter is firstly designed according to the parameters provided by the synthesis defined above. To meet the requirements of the coupling matrix, there are some design rules to follow. To begin with, the diagonal matrix terms are adjusted by the resonator lengths. Then, the off-diagonal terms are set up by the gap between each resonator. Finally, owing to the strong input/output couplings, it is not possible to perform them by proximity. The solution is then to couple them directly with the feeding lines. Their positions determine the values of the couplings but they also create frequency shifts. To counter-balance these effects, a capacitive effect has to be added to each resonator. This is achieved by adding a short length line at the end of each one.

Because of parasitic couplings, filters are firstly tuned by fitting EM responses with the objective curves extracted from coupling matrix consigned in (9) and (10). To take into account the capacitive and inductive effect of the meanders and corners in the junctions, as well as the variation on the line impedance due to the electric walls, each filter was tuned separately but inside the duplexed structure with the initial 


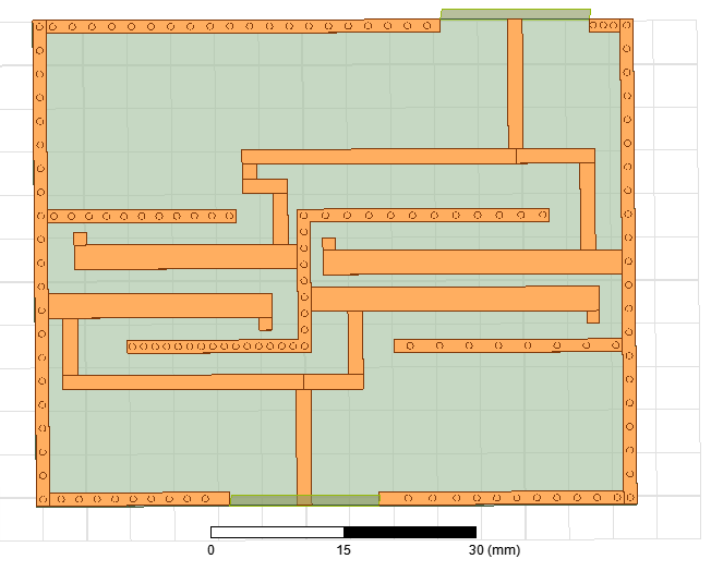

Fig. 8. Layout of the dual-band filter.
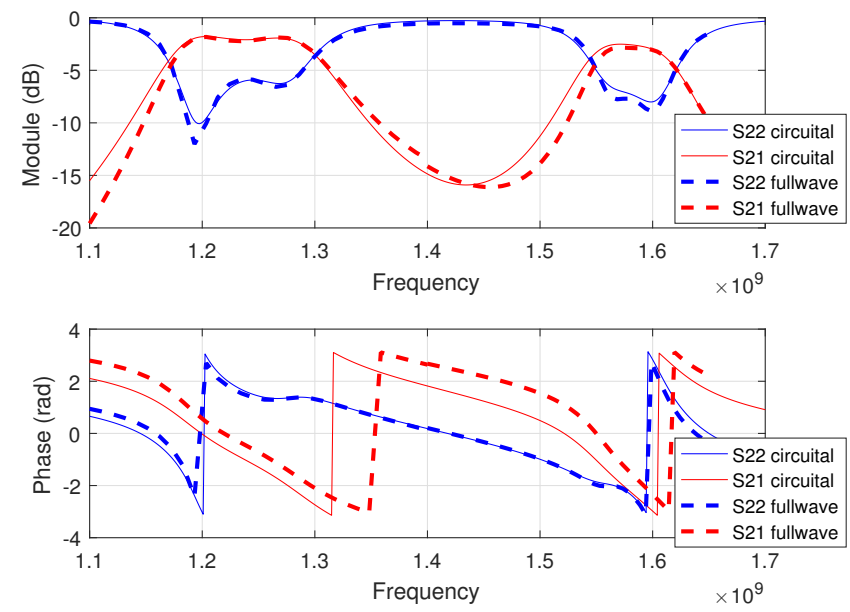

Fig. 9. Response of the dual-band microstrip equalizer.

guess for the length of the transmission lines. The output ports of both filters are then connected together to obtain a diplexer as shown in Fig. 7b. Slight adjustments were necessary to adjust the response of the physical structure to fit the ideal one (whether it be with radiated losses or with return losses) but also to correct the phase with some line lengths corrections (Tr. line 1 and Tr. line 2). The last step is to compute the line lengths at the input of each filter ( $T r$. line 3 and $T r$. line 4). These lengths are deduced from the EM response of the diplexer by looking at the phase of the reflection provided by each channel in the other band.

\section{RESULTS}

The results obtained by the simulation are shown in Fig. 9 compared with the theoretical ones. The full-wave simulation response fits well with the one calculated by the mathematical synthesis, both in magnitude and phase in spite of a small variation on the phase of the transmission coefficient that has no influence on the result. After connecting the antenna, we
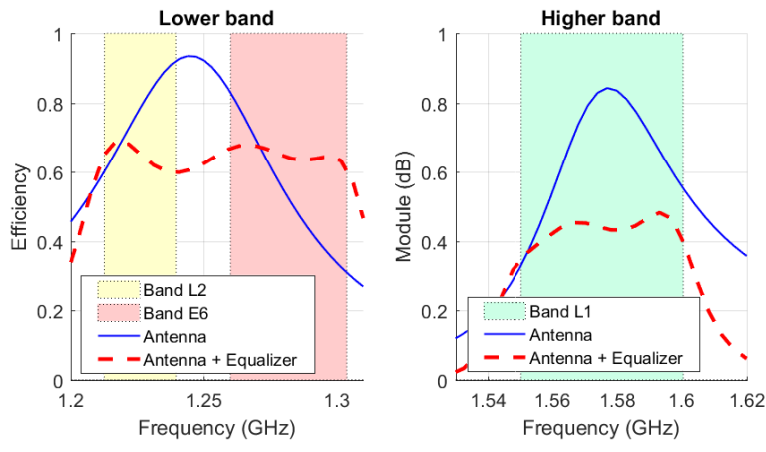

Fig. 10. Obtained efficiency vs original.

obtain the result shown in Fig. 10. It can be seen that, although the efficiency level in the passbands (L2, E6 and L1) is flatter, the level is lower. This is due to the losses in the equalizer filters and should not be seen as a drawback since the received signal must be filter in any case and here the filtering is done by the equalizer.

\section{ACKNOWLEDGEMENT}

This work is performed in the frame of a public research program (ANR ASTRID COCORAM).

\section{CONCLUSION}

A dual-band equalizer was build to improve the total efficiency of a dual-band RHCP antenna. The minimum efficiency of the antenna (at the upper cut-off frequency of the E6 band) was improved from $36 \%$ to (-) \%. Moreover, since the equalizer is composed of two channel filters matched to the antenna, a filtering chain is no longer required with the advantage in size that this entails.

Finally, a prototype has been designed in microstrip technology to prove the feasibility of this approach, obtaining a response that agrees perfectly with the circuital model and provides the expected result in terms of efficiency.

\section{REFERENCES}

[1] M. Troubat, S. Bila, M. Thevenot, D. Baillargeat, T. Monediere, S. Verdeyme, and B. Jecko, "Mutual synthesis of combined microwave circuits applied to the design of a filter-antenna subsystem," IEEE Transactions on Microwave Theory and Techniques, vol. 55, no. 6, pp. 1182-1189, June 2007.

[2] V. Armengaud, J. Lintignat, B. Barelaud, B. Jarry, L. I. Babak, and C. Laporte, "Design of a ka-band mmic filtering lna with a metamorphic hemt technology for a space application," in Microwave Conference, 2008. EuMC 2008. 38th European, Oct 2008, pp. 1358-1361.

[3] N. Demirel, Y. Pinto, C. Calvez, D. Titz, C. Luxey, C. Person, D. Gloria, D. Belot, D. Pache, and E. Kerhervé, "Codesign of a pa-antenna block in silicon technology for 80-ghz radar application," IEEE Transactions on Circuits and Systems II: Express Briefs, vol. 60, no. 4, pp. 177-181, April 2013.

[4] F. Fezai, A. A. Nour, J. Sence, T. Monediere, F. Torres, R. Chantalat, S. Bila, and B. Jarry, "Low-profile dual-band circularly polarized microstrip antenna for gnss applications," in 2015 9th European Conference on Antennas and Propagation (EuCAP), May 2015, pp. 1-4.

[5] H. Bode, Network Analysis and Feedback Amplifier Design, ser. Bell Telephone Laboratories series. Van Nostrand, 1945. [Online]. Available: https://books.google.fr/books?id=kSYhAAAAMAAJ 
[6] R. Fano, Theoretical Limitations on the Broadband Matching of Arbitrary Impedances, ser. Technical report: Research Laboratory of Electronics. MIT Res. Lab. of Electronics, 1947. [Online]. Available: https://books.google.fr/books?id=i1MBHQAACAAJ 